\title{
INTEGRABILITY AND BOUNDEDNESS OF EXTREMAL FUNCTIONS OF A HARDY-SOBOLEV TYPE INEQUALITY
}

\begin{abstract}
YUTIAN LEI
Abstract. In this paper, we study the properties of positive solutions of an integral equation in $R^{n}$$$
u(x)=\int_{R^{n}} \frac{u^{\gamma}(y) d y}{|x-y|^{n-\alpha}|y|^{-\sigma}}, \quad x \in R^{n} .
$$

Such a nonlinear singular equation is related to the study of the best constant of the Hardy-Sobolev type inequality. According to the Newton potential theory, this integral equation is helpful to understand the Henon type partial differential equation when $\alpha=2$. We use the weighted Hardy-Littlewood-Sobolev inequality to obtain the optimal integrability interval of positive integrable solutions. Namely, if $u \in L^{\frac{2 n}{n-\alpha-\sigma}}\left(R^{n}\right)$, then $u \in L^{t}\left(R^{n}\right)$ for all $t>\frac{n}{n-\alpha-\sigma}$. Based on this result, we prove that those integrable solutions must be bounded.
\end{abstract}

Mathematics subject classification (2010): 26D15, 45E10, 45G05.

Keywords and phrases: Hardy-Sobolev type inequality, extremal functions, integrability interval, weighted Hardy-Littlewood-Sobolev inequality.

\section{REFERENCES}

[1] M. Bidaut-Veron, V. Galaktionov, P. Grillot, L. Veron, Singularities for a 2-dimensional semilinear elliptic equation with a non-Lipschitz nonlinearity, J. Differential Equations, 154 (1999), $318-338$.

[2] J. BYeOn, Z. WANG, On the Henon equation: Asymptotic profile of ground states. II, J. Differential Equations, 216 (2005), 78-108.

[3] L. CAFfarelli, R. Kohn, L. Nirenberg, First order interpolation inequalities with weights, Compos. Math. 53 (1984), 259-275.

[4] M. Calanchi, B. RUF, Radial and non radial solutions for Hardy-Henon type elliptic systems, Calc. Var. Partial Differential Equations, 38 (2010), 111-133.

[5] B. GIDAS, J. SPRUCK, Global and local behavior of positive solutions of nonlinear elliptic equations, Comm. Pure Appl. Math., 34 (1981), 525-598.

[6] M. Henon, Numerical experiments on the stability of spherical stellar systems, Astronomy and astrophysics, 24 (1973), 229-238.

[7] C. Jin, C. LI, Symmetry of solutions to some systems of integral equations, Proc. Amer. Math. Soc., 134 (2006), 1661-1670.

[8] C. JIN, C. LI, Qualitative analysis of some systems of integral equations, Calc. Var. Partial Differential Equations, 26 (2006), 447-457.

[9] Y. LEI, Asymptotic properties of positive solutions of the Hardy-Sobolev type equations, J. Differential Equations, 254 (2013), 1774-1799.

[10] Y. LEI, C. LI, C. MA, Asymptotic radial symmetry and growth estimates of positive solutions to weighted Hardy-Littlewood-Sobolev system, Calc. Var. Partial Differential Equations, 45 (2012), $43-$ 61.

[11] E. LiEB, Sharp constants in the Hardy-Littlewood-Sobolev and related inequalities, Ann. of Math., 118 (1983), 349-374.

[12] E. Lieb AND M. Loss, Analysis, 2nd edition, American Mathematical Society, Rhode Island, 2001. 
[13] G. LU, J. ZHU, Symmetry and regularity of extremals of an integral equation related to the HardySobolev inequality, Calc. Var. Partial Differential Equations, 42 (2011), 563-577.

[14] Q. Phan, P. Souplet, Liouville-type theorems and bounds of solutions of Hardy-Henon equations, J. Differential Equations, 252 (2012), 2544-2562.

[15] E. M. Stein, G. Weiss, Fractional integrals in n-dimensional Euclidean space, J. Math. Mech., 7 (1958), 503-514.

[16] Y. ZHAO, Y. LEI, Asymptotic behavior of positive solutions of a nonlinear integral system, Nonlinear Anal., 75 (2012), 1989-1999. 